\title{
Enhanced Spontaneous Raman Signal Collected Evanescently by Silicon Nitride Slot Waveguides
}

\author{
Ashim Dhakal, Frédéric Peyskens, Ananth Z. Subramanian, Nicolas Le Thomas and Roel Baets \\ Photonics Research Group, INTEC Department-Gent University-imec, \\ Center for Nano- and Biophotonics, \\ Ghent University, 9000 Gent, Belgium \\ Authore-mail address: $\underline{\text { ashim.dhakal@intec.ugent.be }}$
}

\begin{abstract}
We investigate the effect of waveguide geometry on the conversion efficiency of Raman signals collected by integrated photonic waveguides. Compared to strip-type photonic wires, we report a six-fold increase in conversion efficiency for silicon-nitride slot-waveguides. OCIS codes: (130.0130) Integrated Optics; (170.5660) Raman spectroscopy
\end{abstract}

\begin{abstract}
1. Introduction
Raman spectroscopy is a direct technique for detection and analysis of chemical and biological substances [1]. However, because of the extremely small cross section of the Raman scattering process, usually a high-power pump laser, sensitive detectors and longer detection time are required. This limits the widespread use of this valuable technique. While most of the existing Raman spectroscopy systems use a confocal microscope, a fiber probe or a hollow fiber, we recently proposed and demonstrated a lab-on-a-chip approach to Raman spectroscopy based on single mode nano-photonic waveguides [2]. In this approach, the molecules under study are evanescently excited and the corresponding Raman signal is also evanescently collected using the same single-mode photonic waveguides. This technique exploits the enhancement effects inherent to the high-index-contrast waveguide and a long interaction length, which as per our calculations, leads to at least two orders of magnitude higher signal than in confocal microscopes. Since a single waveguide carries the pump beam and also collects the Stokes light, it can be easily integrated on a chip with additional components, such as an Arrayed Waveguide Grating (AWGs) acting as an on-chip spectrometer, facilitating development of compact, low-cost systems with high performance [3]. Further, these nano-photonic chips can be mass-produced using CMOS-compatible process steps, which may lead to a disposable, point-of-need tool for Raman analysis of chemical and biological materials. In this paper, we investigate the effect of waveguide width for strip and slotted waveguide geometry on the conversion efficiency of these Raman sensors, which is defined as the ratio between the emitted power collected by the waveguide and the incident pump power in the mode.
\end{abstract}

\section{Methodology and corresponding results}

The conversion from a guided pump beam to a guided Stokes signal can be expressed, for guided modes in awaveguide of arbitrary geometry, by a unit-less parameter, called specific conversion efficiency $\eta_{0}$ defined as [2]

$$
\eta_{0} \equiv \iint_{\text {analyse }} \frac{d \vec{r}}{n}\left(\frac{\pi \kappa_{v} \lambda_{0} n_{g}|E(\vec{r})|^{2}}{\iint \varepsilon_{0} \varepsilon(\vec{r})|E(\vec{r})|^{2} d \vec{r}}\right)^{2}
$$

In this expression $n_{g}$ is the group index of the mode, $\lambda_{0}$ is the wavelength of pump and the Raman signal (the Stokes shift is neglected since its effect on conversion efficiency is small) and $\kappa_{v}$ is a constant with a value of $1.26 \times 10^{23} \mathrm{~V}^{2} \mathrm{~m}^{2} / \mathrm{C}^{2}$ used to convert the Raman polarizability to the cross-section [1]. In eq. (1), the external integral is carried out in the region of refractive index $n$ where analyte molecules are uniformly distributed; the internal integral is carried out in the whole space. The conversion efficiency $(\eta)$ for an infinitesimal length $d z$ of the waveguide can then be calculated as $\eta \equiv d P_{\text {wg }} / P_{\text {pump }}=d z \rho \sigma \eta_{0}$ [2], where $\rho$ and $\sigma$ are respectively the density and Raman crosssection of the molecules. Eq. (1) can be solved using mode solvers for waveguides of an arbitrary geometry. Fig. 1 shows $\eta_{0}$ for silicon nitride (refractive index $=1.89$, height of the silicon nitride is fixed at $h=220 \mathrm{~nm}$ ) strip and slot waveguides (fixed slot width, $s=150 \mathrm{~nm}$, waveguide width $w$ for slot discussed throughout this paper is inclusive of the slot width) for various widths calculated using COMSOL finite element mode-solver for $\lambda_{0}=785 \mathrm{~nm}$. In Fig. 1 we clearly see that $\eta_{0}$ decreases as waveguide width is increased, because the confinement of the mode in the core is increased thereby reducing the interaction volume in the upper cladding region where the analyte molecules are located. In particular, we theoretically expect about 6.4 fold increment in $\eta_{0}$ for slot waveguide (slot width $150 \mathrm{~nm}$ ) 
compared to strip waveguide of width $700 \mathrm{~nm}$.

The experimental setup is described in [2]. Essentially, a laser pump at $785 \mathrm{~nm}$ is coupled into the TE mode of the waveguide. Isopropyl Alcohol (IPA) droplets are applied on top of the waveguides as analyte to compare the Raman signal strength for several waveguide geometries. The light transmitted through the waveguide is then collected, filtered and measured using a commercial spectrometer or power meter. The input laser power is set at $70 \mathrm{~mW}$, with an estimated 8dB coupling loss per chip-facet. For experiments, strip waveguides of width $w=550 \mathrm{~nm}$ and $700 \mathrm{~nm}$ are chosen while for slot waveguides, slot width $s=150 \mathrm{~nm}$, and total waveguide width $w=700$ and $w=800$ are chosen. The waveguides are wound as spirals (typical size: $800 \mu \mathrm{m}$ x $500 \mu \mathrm{m}$ ). The Raman spectra were extracted as explained in [2]. The IPA peak corresponding to the C-C-O vibration at $819 / \mathrm{cm}$ wavenumbers is used as reference to compare the values of $\eta_{0}$ for several waveguides. The ratio of total counts per second corresponding to this peak (which quantifies the collected power $\left.P_{c o l}\right)$, and the corresponding transmitted power at the output $\left(P_{t x}\right)$ provides the quasilinear dependencee $\zeta(L)$ on waveguide length $L[2]$,

$\zeta(L) \equiv \frac{P_{c o l}(L)}{P_{t x}(L)}=\rho \sigma \eta_{0}\left[\frac{e^{\Delta \alpha L}-1}{\Delta \alpha}\right] \approx \rho \sigma \eta_{0} L$

Where, $\Delta \alpha$ is the difference between waveguide losses for transmitted pump power and the Raman signal. The measured function $\zeta(L)$ is then fitted with least-mean-squared algorithm to extract the value of $\rho \sigma \eta_{0}$ in the units of counts $/ \mathrm{mW} / \mathrm{s}$ which can be used to compare the value of $\eta_{0}$ for several waveguide geometries. The experimental data and the corresponding values of $\rho \sigma \eta_{0}$ obtained for several waveguides are provided in Fig. 1, which correspond reasonably well with trend predicted theoretically. In particular, in line with the theoretical prediction, we experimentally observe an enhancement of $\eta_{0}$ by a factor of 5.8 for slot waveguide (slot width $150 \mathrm{~nm}$ ) compared to strip waveguide of width $700 \mathrm{~nm}$.
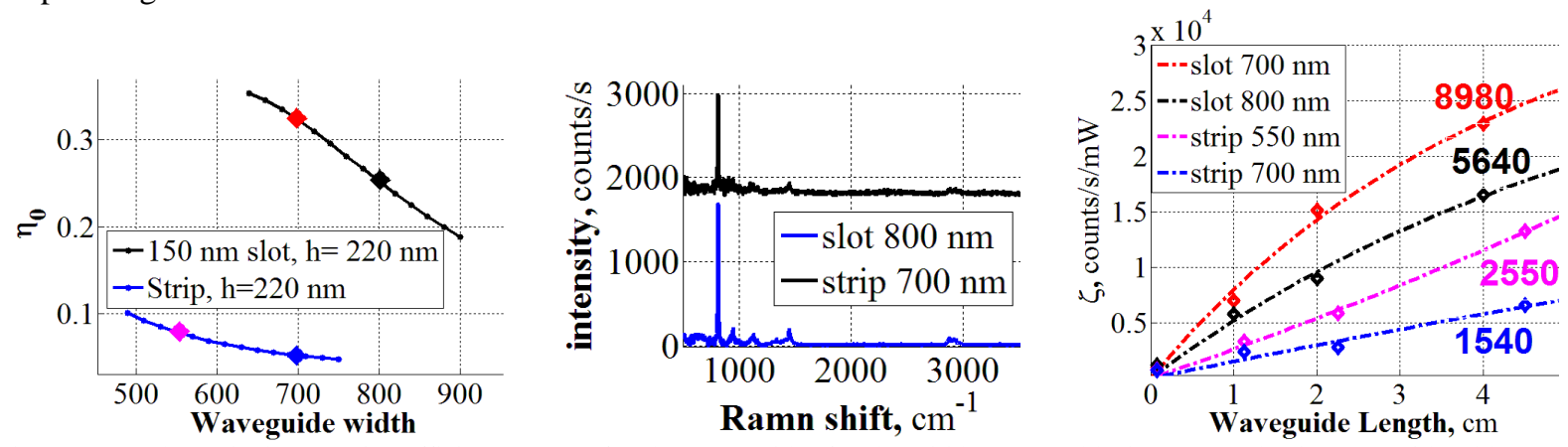

Fig. 1: (Left) Theoretical conversion efficiency curves for slot waveguides for slot width $150 \mathrm{~nm}$ (black curve) and strip waveguides (blue curve). The blue, magenta, red and black diamond shapes are the theoretical values for the waveguides also studied experimentally. (Middle) The spectra of IPA as collected using $800 \mu \mathrm{m}$ long waveguides for slot and strip waveguides. Short waveguide lengths with identical tapers are chosen so that waveguide losses are identical. The top spectrum is shifted vertically for clarity. (Right) The blue, magenta, red and black diamonds are experimental values; while the corresponding quasi-linear lines are the mean squared fit to $\zeta(L)$ as per eq. (2), provided as a guide to the eye. The corresponding extracted values of the $\rho \sigma \eta_{0}$ (in units of counts $/ \mathrm{mW} / \mathrm{s}$ ) are also displayed next to the lines.

\section{Conclusions}

We have theoretically and experimentally investigated the effect of waveguide geometry on the evanescently excited and collected Raman signal using single mode photonic waveguides. We have shown that, in general, slot waveguides have higher conversion efficiency than the strip waveguides. For a given geometry, mode confinement by increasing the waveguide width, leads to smaller conversion efficiency. In particular, we have shown that compared to silicon nitride waveguides with strip geometry, silicon nitride slot waveguide have about six times higher intrinsic conversion efficiency.

\section{References}

[1] D. A. Long, "The Raman Effect: A Unified Treatment of the Theory of Raman Scattering by Molecules", J Wiley \& Sons, Ltd, Chichester, UK (2002)

[2] A. Dhakal, et.al, "Evanescent excitation and collection of spontaneous Raman spectra using silicon nitride nanophotonic waveguides", Optics Letters, 39(13), p.4025-4028 (2014)

[3] R. Baets, et. al., "Resonant enhancement mechanisms in lab-on-chip raman spectroscopy on a silicon nitride waveguide platform, IEEE Photonics Conference 2014 (IPC) United States, p.500-501 (2014) 